\title{
AVALIAÇÃO DE SOBRAS ALIMENTARES EM UMA UNIDADE DE ALIMENTAÇÃO E NUTRIÇÃO INSTITUCIONAL ${ }^{1}$
}

\author{
EVALUATION OF CLEAN FOOD LEFTOVERS AT AN \\ INSTITUTIONAL FOOD AND NUTRITION UNIT
}

\section{Giullia Monteiro Santos ${ }^{2}$, Pietro Marchiori Teixeira Pinto ${ }^{2}$ e Fernanda Ferreira Corrêa ${ }^{3}$}

\section{RESUMO}

Unidades de Alimentação e Nutrição (UANs) desempenham atividades que as tornam grandes produtoras de resíduos, principalmente, de origem orgânica, como sobras alimentares, que são refeições preparadas e não consumidas, por motivos como baixa aceitação ou excesso de produção. O objetivo deste estudo foi avaliar quantitativamente o desperdício de alimentos através de sobras alimentares de preparações sem acréscimo de sal em uma UAN institucional em São Bernardo do Campo (SP), que oferece em média 1200 refeições/dia, com serviço self-service total. Houve um período de cinco dias de pesagem das preparações em questão e de suas respectivas sobras alimentares, no qual foi possível calcular através dos dados obtidos a quantidade final de sobras, a quantidade de alimento que os clientes se serviram e o número de pessoas que seria possível alimentar com a quantidade de sobras descartadas ao final dos cinco dias. A quantidade de sobras foi comparada com a literatura em gramas por pessoa e em porcentagem. Encontrou-se que as sobras alimentares estiveram, durante todos os dias, extremamente acima do recomendado, evidenciando problemas de baixa aceitabilidade por parte dos clientes e consequente desperdício das preparações em estudo, assim como gastos desnecessários gerados na produção das mesmas. Encontrou-se, também, que seria possível alimentar 159 pessoas com a quantidade de sobras durante os cinco dias avaliados. Concluiu-se que estes dados podem servir como respaldo para os nutricionistas responsáveis tomarem medidas corretivas em relação ao desperdício e aos gastos desnecessários.

Palavras-chave: Desperdício de alimentos, resíduos orgânicos, custos.

\section{ABSTRACT}

Food and Nutrition Unities (FNUs) perform activities that turn them into great producers of garbage, mainly organics, such as clean food leftovers, which characterize prepared but not consumed meals, due to nonacceptance or excessive production. This study aimed to quantitatively evaluate the food waste caused through clean food leftovers, prepared without salt, in an institutional FNU in São Bernardo do Campo (SP), which offers in average 1200 meals/day, with total self-service modality. There was a five day weighing period of the mentioned preparations so as its respective leftovers, on which was possible to calculate through the obtained data the final quantity of clean leftovers, the quantity of food that the customers self-served and the number of people that would be possible to feed with the discarded leftovers at the end of the five days. The amount of leftovers was compared with the literature, in grams per person and in percentage. It was found that the clean leftovers were, during all days, extremely above the recommended, indicating problems of low acceptability by the customers and consequent waste of the preparations studied, as well as unnecessary

\footnotetext{
${ }^{1}$ Estudo oruindo de conclusão de Estágio Curricular em Alimentação Institucional.

${ }^{2}$ Acadêmicos do Curso de Nutrição do Centro Universitário São Camilo. E-mail: giullia.smonteiro@gmail.com; pietromarchiori1@hotmail.com

${ }^{3}$ Orientadora. Docente do curso de Nutrição do Centro Universitário São Camilo. E-mail: fernanda.correa@prof.saocamilo-sp.br
} 
expenses generated in their production. It was also found that it would be possible to feed 159 people with the amount of leftovers from the five days period. Therefore, these data perform as a support for the nutritionists in charge to plan corrective measures related to the food waste and the unnecessary expenses.

Keywords: Food waste, organic waste, costs.

\section{INTRODUÇÃO}

Unidades de Alimentação e Nutrição (UAN) são unidades de trabalho que desempenham atividades referentes à alimentação e nutrição, por exemplo, a preparação e o fornecimento de refeições a uma determinada clientela. Seu principal objetivo é oferecer uma alimentação segura, de forma que possa garantir a nutrição necessária para todos aqueles que fazem uso do serviço (BARBOSA et al., 2014).

As UANs acabam por ser grandes produtoras de resíduos, principalmente no decorrer das etapas de produção e distribuição de refeições, sendo a maioria destes resíduos de origem orgânica que podem ter diversas fontes produtoras: compostos gerados no pré-preparo dos alimentos, restos alimentares descartados pelos clientes e sobras de preparações, por exemplo (CARDOSO, MACHADO, 2019).

Sendo assim, o desperdício de alimentos é um grande fator a se atentar nas UANs, pois, além da possibilidade de estar bastante presente nas mesmas, se trata de uma questão ética, já que o Brasil é um país com milhões de pessoas sem acesso à alimentação adequada, e também econômica, que pode fazer grandes diferenças para a empresa e para a Unidade (KAKITANI et al., 2014). Diferentes causas podem ser responsáveis pelo desperdício de alimentos dentro das UANs, entre elas: previsão de gêneros inadequada, preferências alimentares dos comensais, falta de treinamento da mão de obra em relação à produção e distribuição das preparações e até mesmo temperaturas inadequadas (CARDOSO, MACHADO, 2019).

Sobras alimentares limpas são refeições que são preparadas, porém, não distribuídas, logo, não consumidas, seja por não aceitação ou simples excesso de produção; enquanto sobras alimentares sujas, são preparações disponibilizadas no balcão de distribuição, porém não servidas nos pratos; já restos alimentares correspondem a alimentos que são distribuídos e servidos nos pratos, porém não consumidos (KAKITANI et al., 2014; ISSA et al., 2014). Monitorar, analisar e, se possível, registrar as quantidades de sobras alimentares obtidas nas UANs diariamente é de fundamental importância; este acompanhamento pode servir como respaldo para implantar medidas de controle e redução de desperdício, além de permitir que sejam detectadas práticas que possam gerar aumento dos gastos, influenciando diretamente na otimização da produtividade da unidade (CARDOSO, MACHADO, 2019; FERIGOLO, BUSATO, 2018; STRAPAZZON et al., 2015).

Vaz (2006) defende que é aceitável uma quantidade de sobras de até 3\% ou de 7 a 25 gramas por pessoa; enquanto Abreu, Spinelli e Zanardi (2003) não determinam uma porcentagem ou quantidade padrão, apontam que o ideal é que a unidade observe suas sobras diárias e, com o tempo, estabeleça seus próprios parâmetros. 
Tendo de antemão a observação da quantidade elevada de sobras sujas produzidas por uma UAN institucional localizada na cidade de São Bernardo do Campo - SP e mediante solicitação da nutricionista responsável pela unidade de que fosse realizada uma análise quantitativa em relação à produção das mesmas, uma vez que a empresa estaria arcando com custos potencialmente desnecessários, o presente estudo teve como objetivo avaliar o desperdício de alimentos oriundo de sobras alimentares sujas de preparações elaboradas sem acréscimo de sal.

\section{MATERIAIS E MÉTODOS}

Trata-se de uma pesquisa analítica, observacional, de delineamento transversal e não controlada. A pesquisa foi desenvolvida em uma UAN institucional de médio porte, que oferece em média 1200 refeições por dia, sendo cerca de 850 delas distribuídas no período do almoço, com serviço self-service total, localizada na cidade de São Bernardo do Campo, no estado de São Paulo.

Para avaliação das sobras sujas, as panelas que continham preparações do balcão denominado " $\mathrm{Da}$ Horta" foram pesadas, sendo estas: uma opção de prato principal, uma opção de guarnição, uma opção de sopa, arroz integral e feijão, sendo todas elaboradas sem acréscimo de sal. A coleta de dados ocorreu durante um período de cinco dias, compreendidos em: 2 de março de 2020 (segunda-feira), 3 de março de 2020 (terça-feira), 4 de março de 2020 (quarta-feira), 5 de março de 2020 (quinta-feira) e 6/03/2020 (sexta-feira). Os momentos de pesagem foram antes da distribuição das refeições (10h30) e após o término (13h00). A partir dos dados obtidos através da pesagem, foram realizados os seguintes cálculos:

- Horário das 10h30 (antes da distribuição das refeições): panela contendo teor total da preparação $(\mathrm{kg})$ - panela vazia $(\mathrm{kg})$ = quantidade de alimento distribuído (kg)

- Horário das $13 \mathrm{~h} 00$ (após o término da distribuição das refeições): panela contendo sobras alimentares $(\mathrm{kg})$ - panela vazia $(\mathrm{kg})$ = quantidade de sobras por preparação $(\mathbf{k g})$

- Quantidade de alimento distribuído (kg) - quantidade de sobras alimentares $(\mathrm{kg})=$ quantidade de alimento de que os clientes se serviram

Destaca-se que tanto os cálculos quanto a posterior avaliação foram realizados apenas com a quantidade de alimento transportada ao refeitório para distribuição no balcão. As sobras denominadas limpas permaneciam na cozinha e não foram avaliadas. Para pesagem foi utilizada balança digital eletrônica SF-400 de alta precisão da marca Chefstock ${ }^{\circledR}$.

Através dos resultados da pesagem, a quantidade de sobras foi comparada com a literatura, em gramas por pessoa e em porcentagem, para avaliar se a quantidade jogada fora é excessiva ou não. Para isso seguiram-se os critérios de Vaz (2006): quantidade de sobras de até 3\% ou de 7 a 25 gramas por pessoa foi considerada aceitável. Para analisar o impacto negativo para a empresa através de outros parâmetros, foram tabuladas em gráfico a quantidade total de refeições em cada um dos cinco dias analisados e a quantidade relativa de cada rampa que a unidade dispõe: rampas denominadas 
“Tradicionais", onde o cardápio segue o padrão da unidade, sem quaisquer alterações, "Du Chef”, onde há uma opção especial de prato principal, sempre porcionada por um colaborador e "Da Horta", onde as preparações são feitas sem o acréscimo de sal, sendo a última o alvo de análise do estudo. Os dados foram obtidos por meio do registro diário que a unidade mantém.

Foi realizado o cálculo do número de pessoas que seria possível alimentar com a quantidade de sobras dos cinco dias avaliados. Este cálculo foi feito dividindo a quantidade total de sobras em gramas, pelo peso per capita médio da refeição. O peso per capita médio da refeição foi obtido dividindo a quantidade total de que os clientes se serviram pelo número de refeições.

\section{RESULTADOS E DISCUSSÃO}

Os resultados referentes à quantidade distribuída de alimentos em quilos, a quantidade consumida e a quantidade de sobras alimentares de cada dia e do total dos dias estão apresentados na Tabela 1.

Valores percentuais referentes aos resultados demonstrados na Tabela 1 apontam que: 31\% da quantidade distribuída foi consumida pelos clientes na segunda-feira; $29 \%$ na terça-feira; $45 \%$ na quarta-feira; $43 \%$ na quinta-feira e $45 \%$ na sexta-feira. No total de dias, a quantidade de que os clientes se serviram corresponde a $39 \%$ da quantidade total distribuída.

Em relação à quantidade de sobras alimentares, esta sempre ultrapassa metade da quantidade distribuída, correspondendo a 69\% na segunda-feira; $71 \%$ na terça-feira; 55\% na quarta-feira; $57 \%$ na quinta-feira e $55 \%$ na sexta-feira.

Nos cinco dias avaliados, o total das sobras foi de $34,8 \mathrm{~kg}(61 \%)$, isso corresponde à quantidade total de alimentos descartados pela unidade como resíduos orgânicos, visto que essas sobras alimentares não são reutilizadas.

De acordo com o encontrado, a quantidade diária e total de sobras foi extremamente acima do percentual recomendado de 3\% (VAZ, 2006). Em relação à quantidade de sobras em gramas por pessoa, em nenhum dos dias a quantidade média ficou entre a faixa recomendada de 7 - 25 gramas (VAZ, 2006), mantiveram-se bastante acima do recomendado: $386 \mathrm{~g} /$ pessoa na segunda-feira; $431 \mathrm{~g} / \mathrm{pessoa}$ na terça-feira; 294 g/pessoa na quarta-feira; 329 g/pessoa quinta-feira e 302 g/pessoa na sexta-feira.

Tabela 1 - Quantidade diária e total de alimentos distribuídos, servidos e de sobras alimentares, em quilos.

\begin{tabular}{cccc}
\hline Dias da semana de análise & Quantidade distribuída (Kg) & Quantidade servida (Kg) & Quantidade de sobras (Kg) \\
\hline Segunda-feira & 11,20 & 3,49 & 7,71 \\
\hline Terça-feira & 12,17 & 3,55 & 8,62 \\
Quarta-feira & 10,75 & 4,87 & 5,88 \\
Quinta-feira & 11,63 & 5,05 & 6,58 \\
Sexta-feira & 10,94 & 4,91 & 6,03 \\
\hline TOTAL & 56,7 & 21,9 & 34,8 \\
\hline
\end{tabular}

Fonte: Construção do autor. 
A análise de sobras tem como objetivo avaliar a quantidade desperdiçada devido a erros de planejamento, como: apresentação das preparações, per capita descabido e aceitação do cardápio (BARBARA et al., 2019). Acredita-se que o critério "aceitação do cardápio" seja o principal causador da quantidade excessiva de sobras produzidas na UAN avaliada, o que é possível visualizar mais nitidamente no Gráfico 1.

Rodriguez et al. (2010), obteve em seu estudo, uma porcentagem de sobras de 13\%, que já é considerada acima do aceitável e, segundo o autor, este alto índice serve como indicador de erros na quantidade produzida, causando consequências como gastos desnecessários. A quantidade de sobras está diretamente relacionada ao número de refeições servidas e à margem de segurança adotada pela unidade. Ambos os itens devem ser considerados durante o planejamento do cardápio, para que a quantidade seja adequada segundo a literatura (CARMO, LIMA, 2011), o que não ocorreu na UAN avaliada no presente estudo.

A Tabela 2 apresenta o cardápio ofertado, as quantidades de alimentos distribuídos e de sobras alimentares, durante a semana. É possível assim, avaliar, quais preparações, dentre as comumente ofertadas, são melhores ou piores aceitas pelos clientes. Por exemplo, o dia em que a opção de guarnição foi couve refogada (quarta-feira) e o dia onde o prato principal foi bife grelhado (quinta-feira) não houveram sobras.

Porém, vale ressaltar que o refeitório da unidade possui mais duas opções de rampas diferentes, portanto, o cardápio ofertado nestas duas outras rampas também pode influenciar no consumo da rampa de estudo.

Tabela 2 - Quantidade em quilos distribuída de alimentos e de sobras alimentares por preparação servida.

\begin{tabular}{clcc}
\hline $\begin{array}{c}\text { Dias da semana } \\
\text { de análise }\end{array}$ & \multicolumn{1}{c}{ Cardápio } & $\begin{array}{c}\text { Quantidade distribuída } \\
\text { por alimento (Kg) }\end{array}$ & $\begin{array}{c}\text { Quantidade de sobras } \\
\text { por alimento (Kg) }\end{array}$ \\
\hline \multirow{3}{*}{ Segunda-feira } & Arroz integral sem sal & 2,26 & 1,57 \\
& Feijão sem sal & 2,91 & 2,24 \\
& Sopa de ervilha & 3,01 & 2,31 \\
& Frango grelhado & 1,91 & 1,05 \\
& Espinafre refogado & 1,11 & 0,54 \\
& Arroz integral sem sal & 2,17 & 1,50 \\
\cline { 2 - 3 } Terça-feira & Feijão sem sal & 3,11 & 2,88 \\
& Sopa de mandioca & 2,81 & 2,25 \\
& Carne moída & 2,42 & 1,00 \\
& Abóbora seca ao vapor & 1,66 & 0,99 \\
& Arroz integral sem sal & 2,20 & 1,33 \\
& Feijão sem sal & 3,37 & 2,21 \\
\cline { 2 - 4 } & Sopa carioca (feijão preto + linguiça) & 2,97 & 1,55 \\
& Proteína de soja & 1,80 & 0,79 \\
& Couve refogada & 0,41 & 0
\end{tabular}




\begin{tabular}{lllc} 
& Arroz integral sem sal & 2,02 & 1,35 \\
\cline { 2 - 3 } Quinta-feira & Feijão sem sal & 3,01 & 1,75 \\
\cline { 2 - 3 } & Sopa de lentilha & 3,15 & 2,30 \\
\cline { 2 - 3 } & Bife grelhado & 1,16 & 0 \\
& Abobrinha ao vapor & 2,29 & 1,18 \\
\cline { 2 - 3 } & Arroz integral sem sal & 2,17 & 1,37 \\
\cline { 2 - 3 } & Feijão sem sal & 3,14 & 1,78 \\
& Sopa de aveia com carne & 2,93 & 2,22 \\
& Frango grelhado em cubos & 1,84 & 0,34 \\
& Repolho refogado & 0,86 & 0,32 \\
\hline
\end{tabular}

Fonte: Construção do autor.

A unidade tem a opção de registrar periodicamente as quantidades de sobras para, então, estabelecer, a longo prazo, um parâmetro próprio. Este tipo de registro se torna importante, pois serve como respaldo para futuras tomadas de decisão, como implantação de medidas para reduzir desperdícios ou adequação do planejamento e da quantidade de produção (BARBARA et al., 2019).

Um estudo realizado em uma UAN institucional em Sergipe/SE mostrou que uma alternativa possível para reduzir a quantidade de sobras seria verificar a possibilidade de melhoras no processo de produção, com o objetivo de aumentar a aceitação por parte dos clientes. Há autores que sugerem estratégias para otimizar a elaboração de cardápios, como fazê-los contando com as opiniões dos clientes. Isso geraria, inclusive, benefícios financeiros para a UAN, uma vez que o resultado seria redução do desperdício (BARBARA et al., 2019).

Os colaboradores e responsáveis pela UAN tem conhecimento de que um número menor de clientes consomem os alimentos da rampa "Da Horta". Com o objetivo de não haver uma super produção destinada especificamente a esta rampa, que causaria desperdício exacerbado, a unidade mantém controle do número médio de clientes servidos diariamente, tanto no total quanto separadamente por rampas. É possível visualizar estes dados no Gráfico 1.

Gráfico 1 - Quantidades total e isolada de refeições consumidas por rampa.

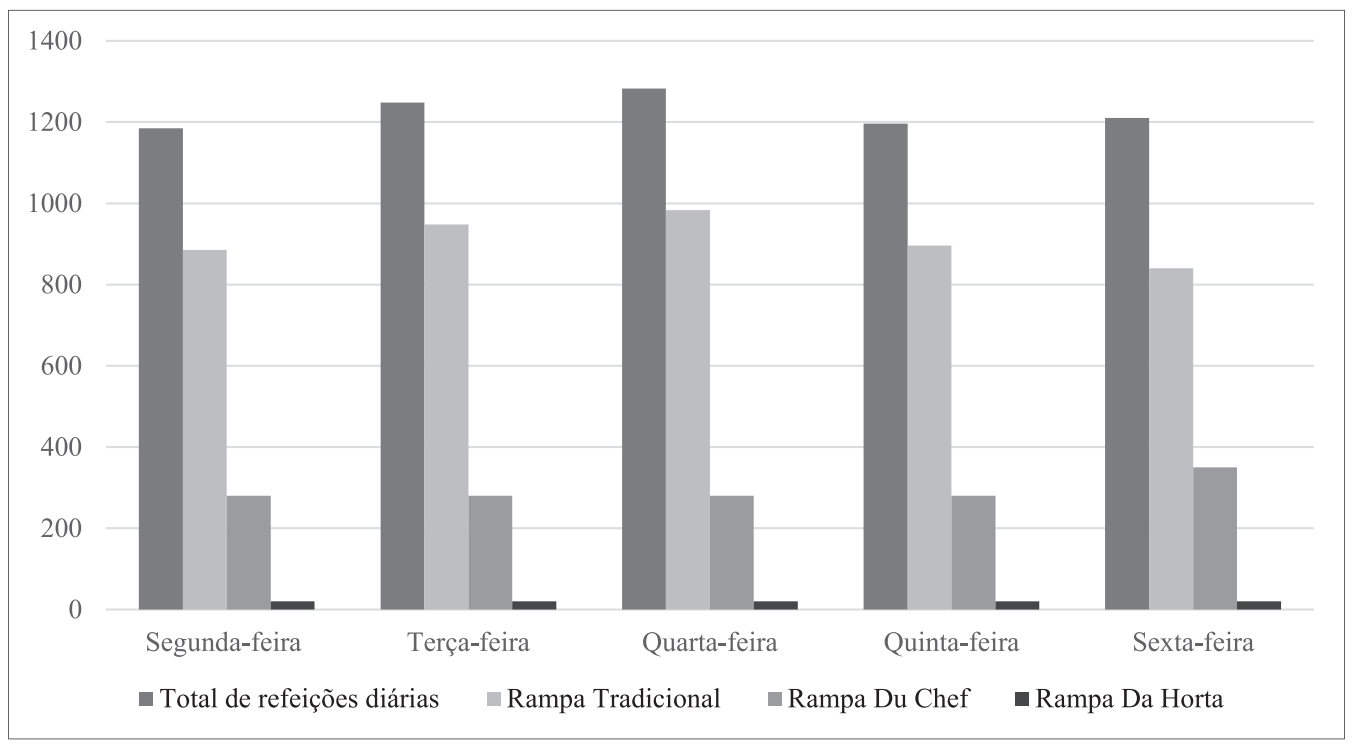

Fonte: Construção do autor. 
Segundo o Gráfico 1, a rampa com maior quantidade de refeições servidas é a Tradicional. A rampa Da Horta manteve uma média de 20 refeições diárias, que representa apenas 1,7\% da média diária de 1200 refeições servidas. Assim, é possível visualizar de maneira mais evidente a frequência de consumo da rampa de estudo, que, em comparação às demais, representa uma parcela bastante pequena.

Ainda que uma menor frequência já seja esperada pela unidade, a quantidade de sobras dos alimentos da rampa avaliada continua sendo acima do considerado aceitável, havendo a necessidade de medidas corretivas.

A percebida baixa aceitação por parte dos clientes pode estar relacionada ao principal fator que contrasta as preparações analisadas com as demais: a isenção do acréscimo de sal. Sabe-se que, atualmente, a população brasileira tende a ingerir quantidades excessivas de sódio, seja através da adição de sal ou através do consumo de produtos industrializados, que normalmente contêm alto teor de sódio (KLEIN, SILVA, 2012). Podendo, assim, estar com o paladar habituado a altas quantidades de sal, identificando refeições preparadas com diferentes temperos como "sem gosto".

A ingestão de sal é considerada um fator relevante no desenvolvimento da hipertensão arterial sistêmica (HAS). Salienta-se, então, a importância de ações educativas em alimentação e nutrição numa UAN, já que a educação nutricional fornece informações que podem servir como auxílio para o indivíduo na tomada de decisões em relação aos seus hábitos alimentares. Cabe ao(s) nutricionista(s) responsável pela unidade que desempenhe campanhas de educação alimentar e nutricional, de maneira contínua, para funcionários e clientes. Promovendo atividades relacionadas ao consumo elevado de sal e suas consequências, por exemplo, de maneira que seu público-alvo esteja devidamente informado (KLEIN, SILVA, 2012). Poderia ser uma alternativa para aumentar a assiduidade no consumo de alimentos do cardápio da rampa de estudo, ainda que a longo prazo. Torna-se importante a conscientização dos clientes acerca dos benefícios que esse padrão de alimentação oferece, podendo optar de maneira autônoma por incluir as refeições em questão em sua rotina.

Durante o período de coleta de dados, estimou-se que seria possível alimentar 159 pessoas com a quantidade acumulada de sobras ao final dos cinco dias de avaliação. É extremamente válido considerar que esta quantidade deva ser reduzida. Estatísticas demonstram que no Brasil, em média, cada pessoa desperdiça cerca de 150 gramas de alimento por dia, totalizando 55 quilos de alimento por pessoa ao final de um ano (RABELO, ALVES, 2016).

Ainda, por um aspecto administrativo, evitar o desperdício impacta positivamente na rentabilidade da UAN, pois as sobras representam preparações que, apesar de não terem sido servidas, trazem uma parcela de custos que se dividem em cada etapa da produção, incluindo assim, custos com matéria-prima alimentar, custos de energia e combustível gastos no preparo e tempo gasto com a mão-de-obra (RABELO, ALVES, 2016), que poderia direcionar seus esforços, por exemplo, em preparações melhor aceitas pelos clientes. 


\section{CONSIDERAÇÕES FINAIS}

Com o objetivo principal de avaliar as sobras obtidas pela rampa "Da Horta", onde as preparações são elaboradas sem acréscimo de sal, encontrou-se que a quantidade total de sobras acumulada ao final de cinco dias de avaliação está acima do recomendado pela literatura.

Estes dados podem servir como respaldo para as nutricionistas responsáveis tomarem medidas corretivas em relação ao desperdício que essa rampa oferta. Isso pode ser em decorrência da parcela mínima de clientes que se serviram no balcão de estudo. Uma sugestão seria retirá-lo do refeitório e redistribuir algumas das preparações mais aceitas nas demais rampas, que costumam ter mais assiduidade por parte dos clientes. Além da possibilidade de implementar ações de educação alimentar e nutricional que abordem sobre o consumo elevado de sal pela população brasileira e seus impactos na saúde, salientando os benefícios que poderiam decorrer da alimentação isenta de acréscimo de sal.

\section{REFERÊNCIAS}

ABREU, E.S.; SPINELLI, M. G. N.; ZANARDI, A. M. P. Gestão de unidades de alimentação e nutrição: um modo de fazer. São Paulo: Ed. Metha, 2003.

BARBARA, D. S. et al. Avaliação de sobras limpas e resto-ingesta em uma unidade de alimentação e nutrição na cidade de Nossa Senhora do Socorro-SE. Revista Ciência (In) Cena, Salvador, v. 2, n. 9 , p. 52-59, 2019.

BARBOSA, V. P. et al. A. Diagnóstico do desperdício de alimentos em uma unidade de alimentação e nutrição institucional em Confidentes-MG. In: Congresso Nacional de Meio Ambiente de Poços de Caldas, 11., 2014, Minas Gerais, Brasil. Diagnóstico [...]. Poços de Caldas: Revista GEINTEC, 2014. p. 3121-3127.

CARDOSO, W. K. L.; MACHADO, C. C. B. Percentual de sobras e resto-ingestão em unidades de alimentação e nutrição institucionais. Revista Saber Científico, Porto Velho, v. 8, n. 1, p. 81-88, jan./jun., 2019.

CARMO, S. O.; LIMA, T. P. Avaliação do índice de sobras limpas em uma Unidade de Alimentação e Nutrição (UAN) institucional na cidade de Campo Grande - MS. Ensaios e Ciência: Ciências Agrárias, Biológicas e da Saúde, [s.l.], v. 15, n. 6, p. 9-20, 2011.

FERIGOLLO, M. C.; BUSATO, M. A. Desperdício de alimentos em unidades de alimentação e nutrição: uma revisão integrativa da literatura. Holos, [s.l.], v. 1, n. 34, p. 91-102, jan., 2018. 
ISSA, R. C. et al. Alimentação escolar: planejamento, produção, distribuição e adequação. Revista Panamericana de Salud Pública, [s.l.], v. 35, n. 2, p. 96-103, 2014.

KAKITANI, R.; SILVA, T. I. F. F.; SHIINO, E. T. Desperdício de alimento no pré-preparo e pós-preparo em um refeitório industrial. Revista Ciências do Ambiente On-Line, [s.l.], v. 10, n. 1, 2014.

KLEIN, C.; SILVA, A.B.G. Avaliação do consumo de sódio em uma unidade de alimentação e nutrição do Vale do Taquari. Revista Destaques Acadêmicos, [s.l.], v. 4, n. 3, p. 73-78, 2012.

RABELO, N. M. L.; ALVES, T. C. U. Avaliação do percentual de resto-ingestão e sobra alimentar em uma unidade de alimentação e nutrição institucional. Revista brasileira de tecnologia agroindustrial, [s.l.], v. 10, n. 1, p. 2039-2052, jan./jun., 2016.

RODRIGUEZ, A. C. et al. Análise do índice de Resto-ingestão e de Sobras de uma UAN localizada no município de São Paulo, SP. Revista Higiene Alimentar, [s.l.], v. 24, n. 184/185, p. 22-24, 2010.

SPEGIORIN, L. A.; MOURA, P. N.. Monitoramento de sobras limpas: um passo para a redução do desperdício em unidades de alimentação e nutrição (UANs). Revista Salus- Guarapuava, Paraná, v. 3, n. 1, p. 15-22, jan./jun., 2009.

STRAPAZZON, J. et al. Sobras e resto ingesta:uma avaliação do desperdício. Nutrição Brasil, [s.l.], v. 14, n. 3, p. 127-131, 2015.

VAZ, C. S. Restaurantes - Controlando custos e aumentando lucros. Brasília: Ed. LGE, 2006. 
